\title{
The combination of nano-calcium sulfate/ platelet rich plasma gel scaffold with BMP2 gene-modified mesenchymal stem cells promotes bone regeneration in rat critical- sized calvarial defects
}

Zunpeng Liu' ${ }^{1,4}$, Xue Yuan ${ }^{1}$, Gabriela Fernandes ${ }^{1}$, Rosemary Dziak' , Ciprian N. Ionita ${ }^{5}$, Chunyi Li', Changdong Wang ${ }^{1}$ and Shuying Yang ${ }^{1,2,3^{*}}$ (i)

\begin{abstract}
Background: Mesenchymal stem cells (MSCS) can be differentiated into an osteoblastic lineage in the presence of growth factors (GFs). Platelet-rich plasma (PRP), which can be easily isolated from whole blood, contains a large amount of GFs, and, therefore, promotes bone growth and regeneration. The main goal of this work was to develop and investigate the effect of a new sandwich-like bone scaffold which combines a nano-calcium sulfate (nCS) disc along with PRP fibrin gel (nCS/PRP) with BMP2-modified MSCs on bone repair and regeneration in rat critical-sized calvarial defects.

Methods: We evaluated the cytotoxicity, osteogenic differentiation and mineralization effect of PRP extract on BMP2-modified MSCs and constructed a sandwich-like nCS/PRP scaffold (mimicking the nano-calcium matrix of bone and carrying multi GFs in the PRP) containing BMP2-modified MSCs. The capacity of this multifunctional bone regeneration system in promoting bone repair was assessed in vivo in a rat critical-sized $(8 \mathrm{~mm})$ calvarial bone defect model.

Results: We developed an optimized nCS/PRP sandwich-like scaffold. Scanning electron microscopy (SEM) results showed that nCS/PRP are polyporous with an average pore diameter of $70-80 \mu \mathrm{m}$ and the cells can survive in the nCS/PRP scaffold. PRP extract dramatically stimulated proliferation and differentiation of BMP2-modified MSCs in vitro. Our in vivo results showed that the combination of BMP2-modified MSCs and nCS/PRP scaffold dramatically increased new bone regeneration compared with the groups without PRP and/or BMP2.
\end{abstract}

Conclusions: nCS/PRP scaffolds containing BMP2-modified MSCs successfully promotes bone regeneration in critical-sized bone defects. This system could ultimately enable clinicians to better reconstruct the craniofacial bone and avoid donor site morbidity for critical-sized bone defects.

Keywords: Bone morphogenetic protein 2, Mesenchymal stem cells, Platelet-rich plasma, Critical-sized bone defect, Bone tissue engineering, Bone scaffolds

\footnotetext{
*Correspondence: shuyingy@upenn.edu

1 Department of Oral Biology, School of Dental Medicine, University of

Buffalo, The State University of New York, Buffalo, NY, USA

${ }^{2}$ Developmental Genomics Group, New York State Center of Excellence in

Bioinformatics and Life Sciences, University of Buffalo, The State University of

New York, Buffalo, NY, USA

Full list of author information is available at the end of the article
} 


\section{Background}

For critical-sized bone defects, conventional treatment methods use autografts, allografts, xenografts, and synthetic bone grafts. Although these grafts have shown satisfactory results of bone regeneration, they often possess disadvantages [1] including donor site morbidity, shortage of autografts, risk of disease transmission and immune rejection $[2,3]$. These disadvantages potentiate a need for developing newer materials as bone grafts that can demonstrate better results, reduce costs, and overcome problems associated with existing grafts.

It has been well known that stem cells, especially mesenchymal stem cells (MSCs), have been used for hard tissue engineering over the years [4-6]. The advantages in the use of MSCs resides in the fact that unlike autogenous grafts, which are not easily available, even a small portion of MSCs derived from tissue can be expanded in in vitro culture for transplantation into defects to advance repair and remodeling of several tissues $[7,8]$. Since these MSCs can be autologous (obtained from the same patient), this decreases the risk of disease transmission and immune rejection.

In order to promote MSC differentiation and proliferation, it is essential to have the presence of a catalyst, which can accelerate this potential without affecting its cellular structure and biology [9], and is inexpensive, biocompatible and osteoconductive $[10,11]$. Bone morphogenetic proteins (BMPs) have been examined for their potential to promote osteoblast proliferation and maturation [12], and are extensively used in bone regeneration [13]. Our previous studies have proved that BMP2 in combination with MSCs is able to repair critical-sized bone defects [14, 15]. Furthermore, BMP2 genetically engineered MSCs have unique advantages for promoting bone regeneration because BMP2 expression is relatively stable, and this treatment is much less expensive than the administration of recombinant BMP2 [14].

Platelet-rich plasma (PRP) consists of several growth factors and the predominate ones are platelet-derived growth factor (PDGF), insulin-like growth factor (IGF), and transforming growth factor beta (TGF-beta) along with their isoforms [16]. PRP also consists of BMP2, which is a powerful stimulator for osteogenic differentiation of MSCs. Upon activation of PRP, these growth factors are released; however, the life span of these growth factors is only 24 hours, which makes it imperative to deliver these growth factors via an agent in order to prolong its function in bone regeneration [17-20].

Recently, nano-calcium sulfate (nCS) has been employed as a scaffold in bone regeneration. It is of enhanced physical properties, such as high surface area for growth factor adsorption, with the potential for controlling the rate of release of the adsorbed material, as well as superior mechanical strength for optimal osteoconductivity and resistance to fractures [21]. Therefore, in this study, we hypothesized that nCS provides good support for PRP, and combined them with BMP2-modified MSCs, and greatly promotes bone formation and regeneration. We found PRP extract significantly promoted the proliferation and osteogenic differentiation of MSCs in vitro. When PRP is combined with nCS and BMP2modified MSCs, it greatly promotes bone formation in a rat critical-sized $(8 \mathrm{~mm})$ calvarial bone defect model.

\section{Methods}

\section{PRP preparation}

PRP and PRP gel were prepared by a modification of the method of Landesberg et al. [22] and have been previously described [23]. Briefly, Sprague-Dawley (SD) rats at 8-week old were anesthetized with tribromoethanol (Avertin, intraperitoneal injection) and the whole blood was collected via retro-orbital puncture. The blood was anticoagulated with $3 \%$ acid-citrate dextrose (1/10 volume) and centrifuged at $200 \times \mathrm{g}$ at $4{ }^{\circ} \mathrm{C}$ for $10 \mathrm{~min}$, then the upper plasma and intermediate layer with fewer red blood cells were collected into a new tube. This new tube was centrifuged again at $200 \times \mathrm{g}$ for $10 \mathrm{~min}$ at $4{ }^{\circ} \mathrm{C}$. The upper layer is called platelet-poor plasma (PPP). Most PPP was removed, and only $0.35 \mathrm{ml} \mathrm{PPP} \mathrm{was} \mathrm{used}$ to resuspend PRP in the bottom. Thrombin (50 U per $1 \mathrm{ml} \mathrm{PRP)} \mathrm{was} \mathrm{added} \mathrm{to} \mathrm{release} \mathrm{the} \mathrm{factors} \mathrm{from} \mathrm{the}$ concentrated platelets and yield PRP gel, which were used for our in vivo experiment (Additional file 1: Figure S1A). PRP gel was centrifuged at $1500 \times \mathrm{g}$ for $5 \mathrm{~min}$, and then the upper solution was harvested as PRP extract. PRP extract contains abundant growth factors and was used immediately after its preparation for our in vitro experiment (Additional file 1: Figure S1A).

\section{Osteogenic differentiation and BMP2 gene transfer}

Rat MSCs were derived from the bone marrow of 6-8week-old SD rats and identified as we described previously [14].

BMP2 adenovirus was produced and titer tested as previously described [14]. Ad-GFP (as a control) or AdBMP2 adenovirus $(\mathrm{MOI}=100)$ was added to MSCs in serum-free medium. After $4 \mathrm{~h}$, serum was added to a final concentration of $2 \%$ for overnight culture. Cells were then switched to osteogenic media containing $50 \mu \mathrm{g} / \mathrm{mL}$ of ascorbic acid, $10 \mathrm{mM} \beta$-glycerophosphate and $10^{-8} \mathrm{M}$ dexamethasone.

\section{Cell proliferation and viability}

MSCs were treated with different concentration of PRP extract $(2.5,5$, and $10 \%)$ for 7 days. At day 1 , day 3 and day 7, cell density was measured by using CellTiter 96 AQueous One Solution Cell Proliferation Assay kit 
(Promega, Madison, WI, USA). Optical density values were measured at $490 \mathrm{~nm}$ using a microplate reader. Three independent biological replicates were assessed.

\section{Alkaline phosphatase (ALP) assay and Alizarin Red staining}

MSCs were induced with osteogenic medium for 7 days for ALP assay and 16 days for Alizarin Red staining. For ALP activity assay, cells were harvested, and the supernatants were used for ALP activity assay as described previously [14]. For Alizarin Red staining, cells were stained with $40 \mathrm{mM}$ of Alizarin Red S solution ( $\mathrm{pH}$ 4.14.4). $10 \%$ cetylpyridinium chloride (in $10 \mathrm{mM}$ sodium phosphate, $\mathrm{pH}$ 7.0) was used to destrain the cells and the optical density (OD) was measured at $562 \mathrm{~nm}$ by an AD 340 microplate reader. Three independent biological replicates were assessed.

\section{Scaffold preparation}

nCS discs were made according to the method of Park et al. [24]. Three-dimensional printed molds (1 $\mathrm{mm}$ thickness $\times 7 \mathrm{~mm}$ diameter) were used to construct the nCS discs. The nCS discs were formulated with nCS and alginate. Alginate was dissolved in PBS to prepare a $10 \%$ solution $(\mathrm{w} / \mathrm{v})$, and then the $\mathrm{pH}$ was adjusted to 7.4-7.6. nCS powder $(135 \mathrm{mg})$ was mixed with alginate solution $(150 \mu \mathrm{l})$. The total mass of $\mathrm{nCS}$ and alginate was $150 \mathrm{mg}$. After $30 \mathrm{~min}$ following the mixing and filling into the $3 \mathrm{D}$ molds with nCS/alginate, the solid nCS discs were formed. Thereafter, the surface of the discs was spread with $40 \mu \mathrm{l} \mathrm{PRP}$, and then $4 \mu \mathrm{l}$ calcium chloride (10\%) was used to convert the PRP to a gel as well as activate the growth factors (Additional file 1: Figure S1B). The PRP gel ( $1 \mathrm{~mm}$ thick) attached to nCS disc to form an nCS/PRP scaffold.

\section{Scanning electron microscopy}

Rat MSCs were seeded on the surface of each nCS/PRP scaffold and cultured for $24 \mathrm{~h}$. Then, the samples were rinsed in $0.1 \mathrm{M}$ phosphate buffer twice and then left in $0.1 \mathrm{M}$ sodium cacodylate (Sigma-Aldrich, St. Louis, MO, USA) for $30 \mathrm{~min}$. The samples were then dehydrated in gradient ethanol and treated with hexamethyldisilizane. The specimens were sputter coated with carbon and observed under a scanning electron microscope.

\section{Rat critical-sized calvarial bone defect model}

The protocol was reviewed and approved by the University at Buffalo Animal Care and Use Committee. Fortytwo male SD rats at 8-week old were used in this study. The animals were divided into seven groups: group 1, blank control (defect only); group 2, nCS only; group 3, nCS + PRP; group 4, nCS (MSCs), group 5, nCS (MSCs) + PRP, group 6, nCS (MSCs/B2) and group 7, $\mathrm{nCS}$
(MSCs/B2) + PRP. The scaffold preparation was showed in Additional file 1: Figure S1B. The surgery process for generation of rat critical-sized calvarial bone defect model and scaffold transplantation were performed as shown in Additional file 1: Figure S1C [14]. Briefly, rats were anesthetized with isoflurane $/ \mathrm{O}_{2}$ gas. The scalps covering the calvarial vault were shaved. An incision was made along the midline of each rat. An 8-mm-diameter trephine bur was used to drill a standardized, round, segmental defect around the sagittal suture. Scaffolds were placed into the defects, and periosteum (pericranium) and skin were sutured. Buprenorphine $(0.05 \mathrm{mg} /$ $\mathrm{kg}), 2 \%$ lidocaine $(20 \mathrm{mg} / \mathrm{ml})$ and carprofen $(5 \mathrm{mg} / \mathrm{kg})$ were given before, during, and after surgery. Eight weeks later, the animals were euthanized using $\mathrm{CO}_{2}$, and calvarial bones were harvested for further analysis.

\section{Analysis of in vivo bone formation}

Bone density measurements (BMD, $\mathrm{g} / \mathrm{cm}^{2}$ ) of all implants from the rats were performed using a LUNAR PIXImus bone densitometer (Lunar Corp., Madison, WI, USA). LUNAR PIXImus software was used to analyze the scanned data. On the computerized scan plots, five regions of interest per implant were selected to measure the BMD of the defect area, and the average values were taken as the final result per mouse. This allowed BMD measurement in close relation to the bone regeneration area by the software excluding pixels.

For histological analysis, half of the calvarial bones were decalcified and cut into $5 \mu \mathrm{m}$ sections. The sections were then stained with hematoxylin and eosin. The ratio of the bone area in the implants against the total implant tissue of section was quantified using $\mathrm{NIH}$ Image J software and defined as the percentage of bone volume in the implant/total volume of the implant.

\section{Micro-CT}

The harvested implants were fixed and scanned using a custom-build micro-CT system $(\mathrm{n}=4$ for each group). The data were analyzed with MicroView 3D Image Viewer \& Analysis Tool (GE Medical Systems, London, ON, Canada).

\section{Statistical analysis}

Statistical analysis was performed using SPSS software version 17.0 (SPSS Inc., Chicago, IL, USA). Experimental data were reported as mean \pm standard deviation of triplicate independent samples. Data were analyzed using Student's $t$ test and one-way analysis of variance, and Tukey's HSD test was applied as a post hoc test if statistical significance was determined. A value of $p \leq 0.05$ was considered statistically significant. 


\section{Results}

PRP promotes BMP2-modified MSCs proliferation and osteogenic differentiation in vitro

PRP has been reported to promote cell proliferation, therefore, we first examined whether PRP could also promote BMP2-modified MSCs proliferation. All groups showed an increase over the 7-day study. At day 7, there was a general increase in cell number with increasing concentration of PRP. The group of $10 \%$ PRP concentration had significantly higher cell proliferation than all other concentration groups (Fig. 1a).

The ALP activity and the level of calcium deposition are important consideration factors for evaluating osteoblast differentiation. MSCs with BMP2 and/or PRP were induced with osteogenic medium for 7 days and then were subjected for ALP activity assay. As expected, BMP2 modification (MSCs + B2) dramatically increased ALP activity ( $p<0.0001$, Fig. $1 \mathrm{~b})$. However, adding $10 \%$ PRP to BMP2-modified MSCs (MSCs + B2 + PRP) could further enhance ALP activity $(p<0.01)$. The level of calcium mineral deposition after 3 weeks in culture was investigated by Alizarin Red staining (Fig. 1c). The quantitative results showed that calcium deposition in the groups of $\mathrm{MSCs}+\mathrm{B} 2+\mathrm{PRP}$ and MSCs + B2 were significantly higher than that in the other groups $(p<$ 0.0001) (Fig. 1d). Adding PRP to BMP2-modified MSCs slightly but not significantly promotes calcium deposition $(p<0.05)$. Furthermore, we studied the structure of nCS and nCS/PRP gel. SEM analysis showed that nCS scaffolds are polyporous with an average pore diameter of $70-80 \mu \mathrm{m}$ (Fig. 2a) and the platelets could attach on the nCS/PRP scaffolds (Fig. 2b).

nCS/PRP gel delivery system with BMP2 genemodified MSCs promotes bone regeneration in vivo. To evaluate the potential of the $\mathrm{nCS} / \mathrm{PRP}$ delivery system and BMP2 gene-modified MSCs for bone in vivo, $8-\mathrm{mm}$ bone defects were created in the calvarial bones of 8week-old SD rats and calvarial bones were harvested at 8 weeks post-surgery. We first examined the bone formation with a BMD test (bone mineral density). The result showed that the $\mathrm{nCS}$ (MSCs/B2) + PRP group exhibited greater bone density than the other groups (Fig. 3a and b), confirming that the combination of $\mathrm{nCS}$ (MSCs/B2) with PRP significantly promotes bone

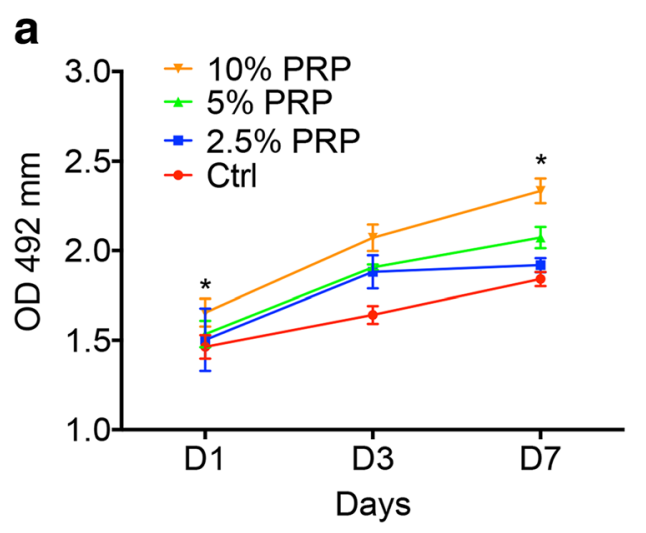

C

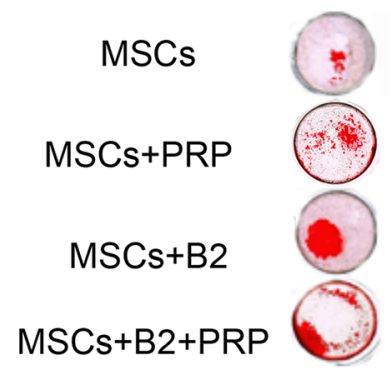

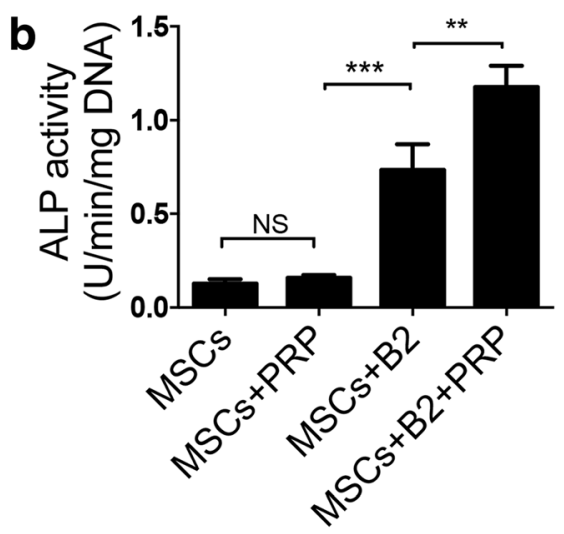

d

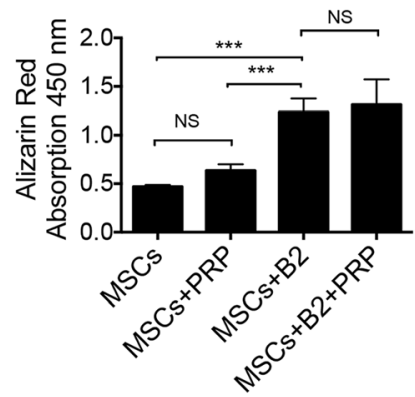

Fig. 1 PRP promotes proliferation and osteogenic differentiation of BMP2-modified MSCs in vitro. a Proliferation of MSCs with different PRP concentrations $(n=4)$. The cell seeding density is $5 \times 10^{4}$ per well in a 96-well plate. MSCs + PRP $(10 \%)$ group has significantly higher cell proliferation rate at days 1,3 , and 7 as compared with other groups and is indicated by ${ }^{*} p<0.05$. b ALP activity of MSCs, MSCs + BMP2 (MSCs + B2), MSCs + PRP, and MSCs + B2 + PRP. The cells were induced with osteogenic media for 7 days. Data represent the mean \pm SD of 3 samples. The ALP activity of MSCs + B2 + PRP group was significantly higher than the other groups as marked as ${ }^{* *} p<0.01 ;{ }^{* * *} p<0.0001 ;$ NS: not statistically significant. c Alizarin Red staining of MSCs, MSCs + B2, MSCs + PRP, and MSCs + B2 + PRP. Cells were cultured in osteogenic medium for 21 days. d Quantitative analysis $(n=5)$ of cell mineralization from Alizarin Red staining shown in $C^{* * *} p<0.0001 ;$ NS: not statistically significant. ALP alkaline phosphatase, B2 bone morphogenetic protein 2, MSCs mesenchymal stem cells, nCS nano-calcium sulfate, PRP platelet-rich plasma 


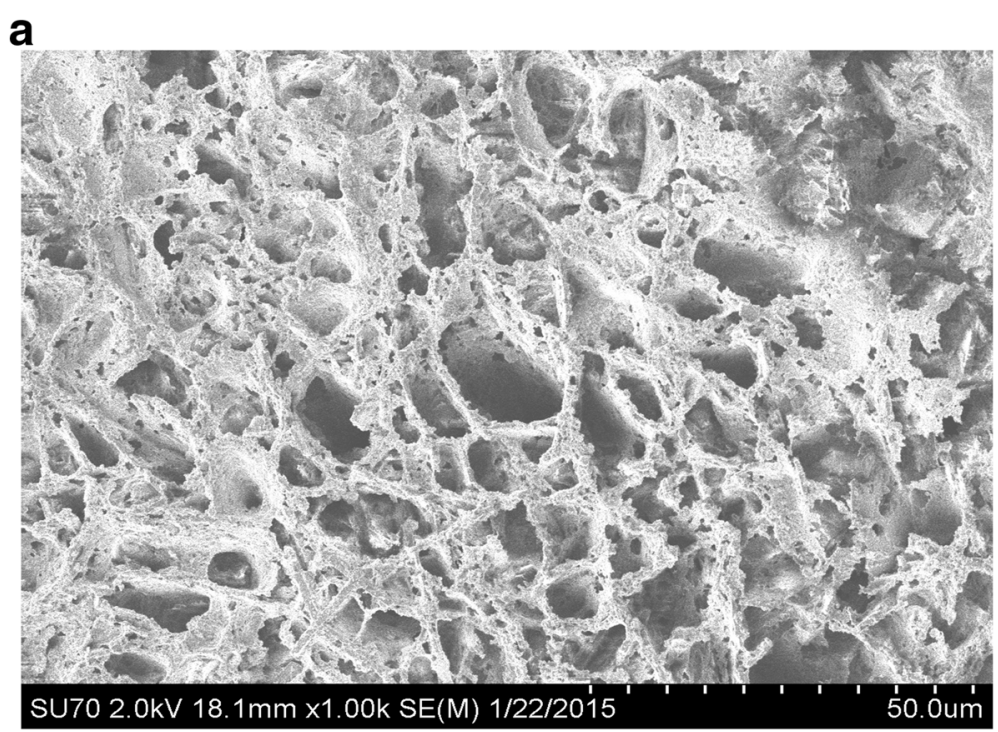

b

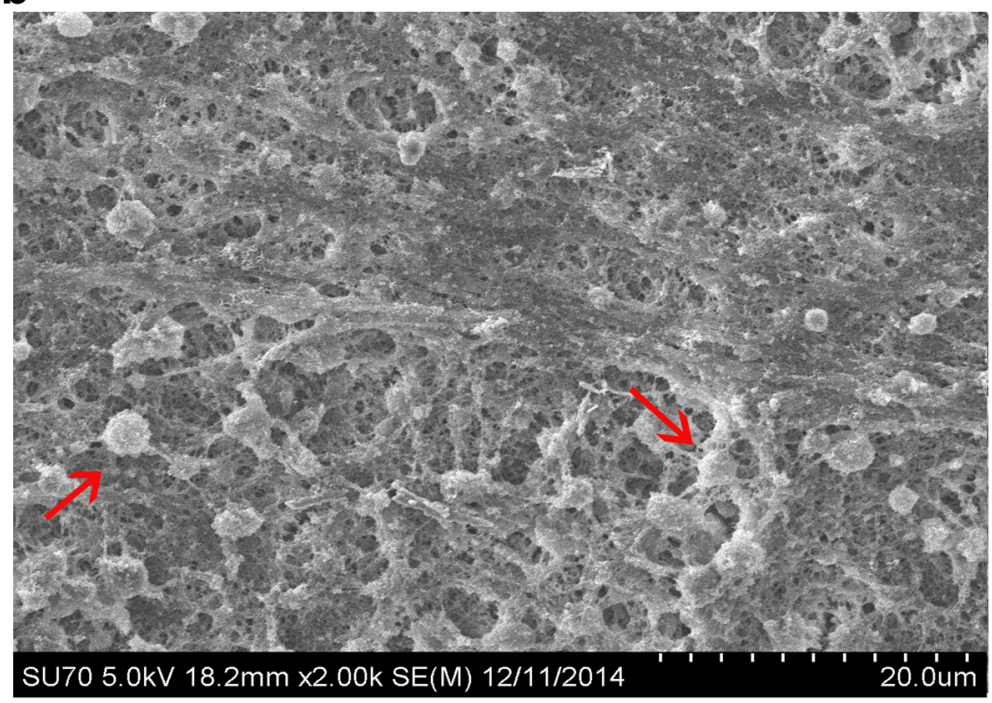

Fig. 2 Scanning electron microscopic (SEM) view of nCS discs (a) and nCS/10\% PRP scaffold (b). Red arrows: platelets

regeneration. The nCS (MSCs/B2) group showed less bone density than $\mathrm{nCS}(\mathrm{MSCs} / \mathrm{B} 2)+\mathrm{PRP}$, however, the bone density is comparable between $\mathrm{nCS}$ (MSCs/B2) and nCS (MSCs) + PRP groups, suggesting PRP is as powerful as BMP2 in promoting bone regeneration (Fig. 3a and b). Similar bone density was shown in the groups of nCS, nCS + PRP, and nCS (MSCs), but those were all higher than the defect-only group (Fig. 3a and b). To further test the bone regeneration activity in different groups, we tested the ALP activity of freshly harvested samples. Consistently, nCS (MSCs/B2), nCS (MSCs) + PRP and nCS (MSCs/B2) + PRP groups showed higher ALP activity compared with other groups, implying more robust osteogenic activity (Fig. 3c). The nCS (MSCs/B2) + PRP group had the highest ALP activity (Fig. 3c), demonstrating that PRP promotes osteogenic differentiation.

Hematoxylin and eosin-stained sections showed that at 8 weeks following the implantation, no residual materials or inflammatory infiltrating cells were seen within any of the defect regions (Fig. 4a and b). In all treatment groups, the smallest amount of new bone was formed in the defect-only group compared with other groups, and most of defect areas were covered by fibrous-like tissues (Fig. 4a and b). The groups of nCS, nCS + PRP, and nCS (MSCs) formed small pieces of new bone, however showed no significant difference, and most of the defect areas were also covered by fibrous-like tissues (Fig. 4a and b). The nCS (MSCs/B2) + PRP group had larger bone formation in the bone defect area compared with 


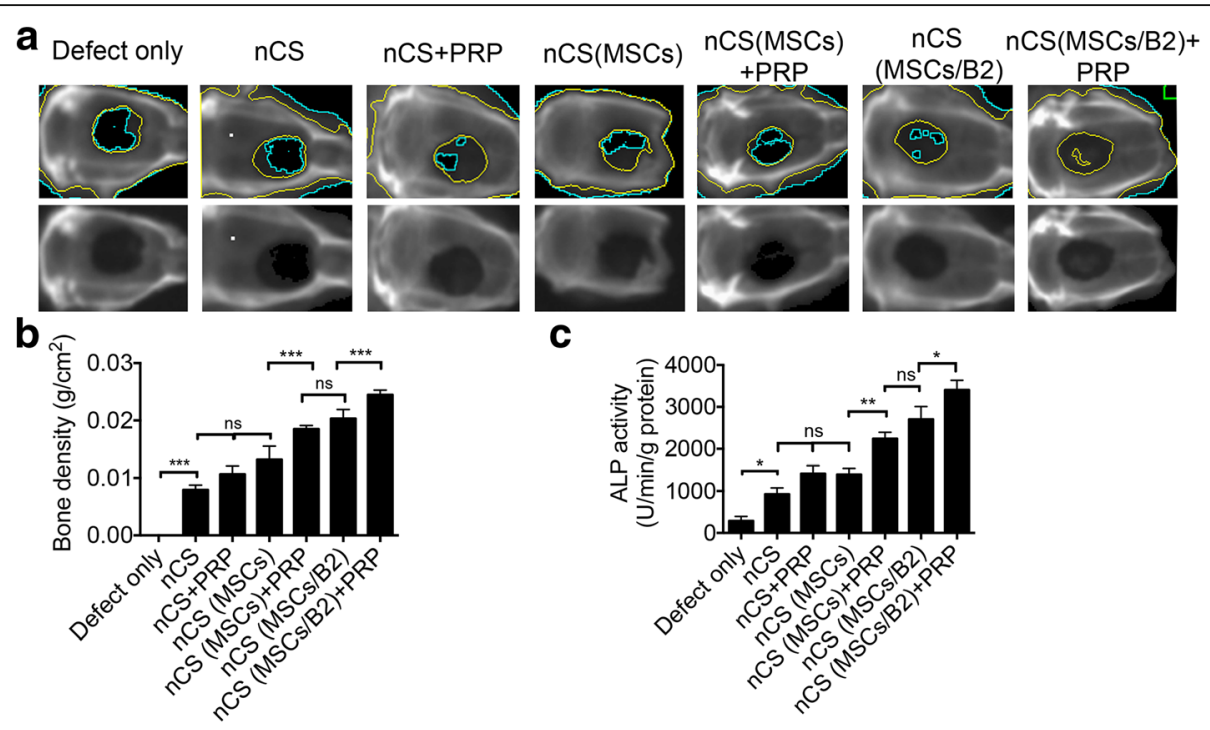

Fig. 3 Bone mineral density analysis. a Images of calvarial bone obtained using LUNAR PIXImus system, 8 weeks after surgery. General X-ray view (lower row) and X-ray with color outlined bone regeneration images (upper row) are showed. The black area circled with green line indicates low density area (fibrous tissue). The gray area between the green line and the yellow lines is the newly-formed bone area. $\mathbf{b}$ Quantitative analysis of bone density from $\mathrm{A}(\mathrm{n}=3)$. c ALP activity of newly formed bone $(\mathrm{n}=3) .{ }^{*} p<0.05 ;{ }^{* *} p<0.01 ;{ }^{* * *} p<0.001$; ns: not statistically significant. ALP alkaline phosphatase, B2 bone morphogenetic protein 2, MSCs mesenchymal stem cells, $n C S$ nano-calcium sulfate, PRP platelet-rich plasma

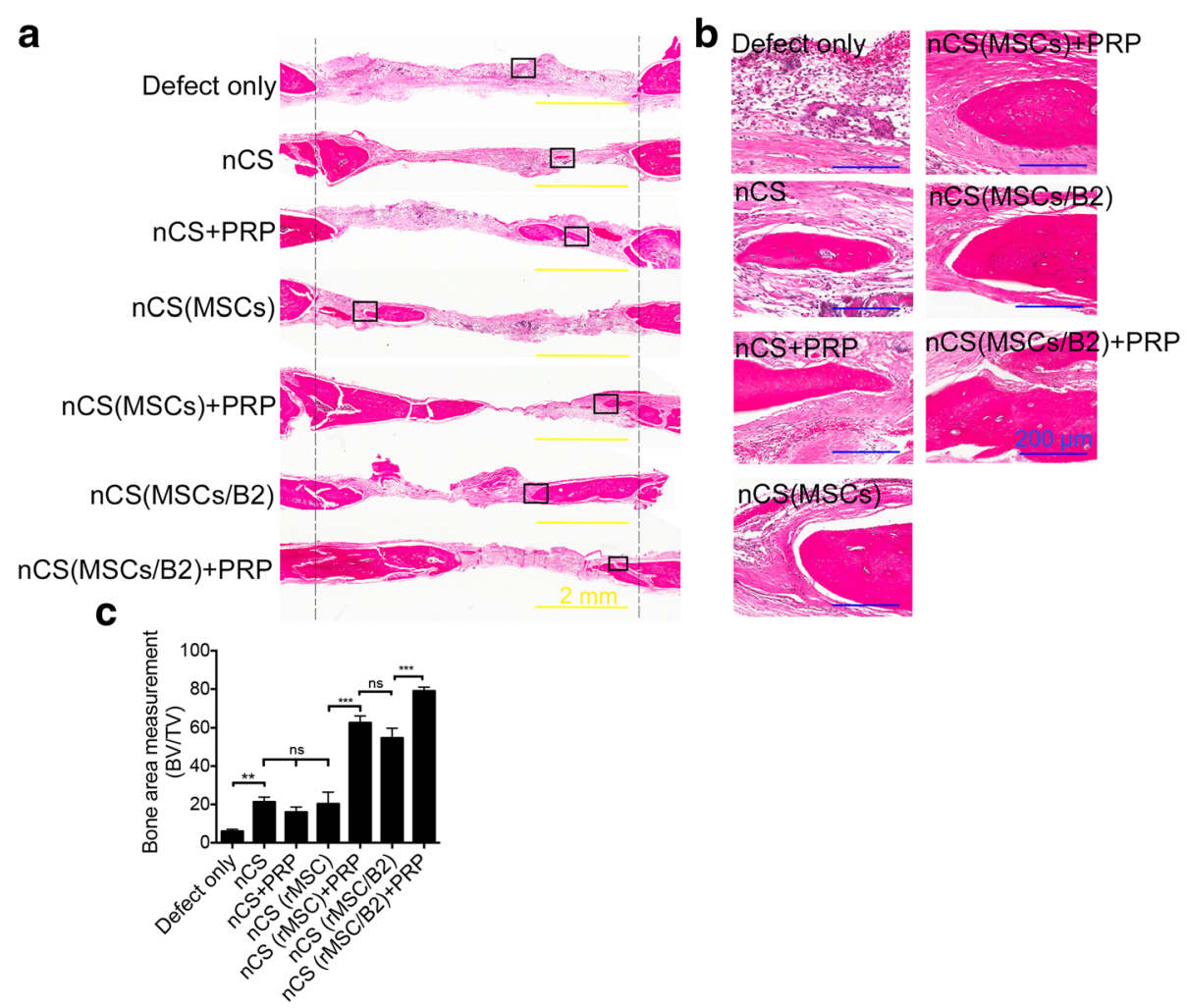

Fig. 4 Histological analysis of newly formed bone. a Hematoxylin and eosin staining of coronal sections through the midline of defects (between dashed line). Combination of nCS/PRP scaffold containing BMP2-modified MSCs promotes new bone formation. Scale bar = $2 \mathrm{~mm}$. $\mathbf{b}$ Higher magnification of hematoxylin and eosin staining shown in A (black rectangle). Scale bar $=200 \mu \mathrm{m}$. c Quantitative analysis of newly formed bone (BV) to the total implant area (TV) $(n=3) .{ }^{* *} p<0.01 ;{ }^{* * *} p<0.001$; $n s$ : not statistically significant. B2 bone morphogenetic protein 2, MSCs mesenchymal stem cells, $n C S$ nano-calcium sulfate, $P R P$ platelet-rich plasma 
nCS (MSCs) + PRP and nCS (MSCs/B2) groups (Fig. 4a and $b$ ), suggesting both BMP2-modified MSCs and PRP are critical for bone formation. Histomorphometric analysis showed that the amount of newly formed bone (BV) to the total implant area (TV) in the nCS (MSCs/ B2) + PRP group was significantly greater than those in the other six groups (Fig. 4c).

Furthermore, we tested the bone regeneration in the defects by micro-CT. As expected, those critical-sized bone defects cannot heal by themselves, therefore, the defect-only group (no treatment) and the scaffold-only groups (nCS) showed less bone regeneration in the defects compared with the groups with MSCs or PRP (Fig. 5a). Moreover, nCS (MSCs/B2) and nCS (MSCs) + PRP have similar new bone formation, suggesting PRP and BMP2 are all important for new bone formation (Fig. 5a). However, PRP combined with MSCs/BMP2 in the $\mathrm{nCS}(\mathrm{MSCs} / \mathrm{B} 2)+\mathrm{PRP}$ group significantly enhanced bone formation, comparing with nCS (MSCs/B2) or nCS (MSCs) + PRP, demonstrating the function of the combination of PRP and BMP2-modified MSCs in inducing bone regeneration (Fig. 5a and b). Quantitative bone volumes in the defect areas analysis demonstrated significantly higher values in nCS (MSCs/B2) + PRP group as compared with the other groups $(p<0.05)$, exhibiting robust osteogenic activity (Fig. 5b).

\section{Discussion}

For improving healing of critical-sized calvarial bone defects, until now, the major concern has been the poor integration of biomaterial scaffold and cells with the neighboring tissue, which slows down bone regeneration. This study, for the first time, investigates the effect of the combination of BMP2-modified MSCs with nCS and
PRP fibrin gel scaffolds on healing critical-sized bone defects. Our results demonstrated the efficiency of this system in bone regeneration, and emphasized the potential ability to employ this system for bone defect treatment.

Calcium sulfate has good biocompatibility, osteoconductivity, and degradation rate for bone regeneration $[25,26]$. As an extension of the study by $\mathrm{He}$ et al. [15], we used nCS along with 10\% PRP fibrin gel as a delivery system to increase the surface area for stem cells attachment and to provide growth factors for promoting cell differentiation and bone regeneration. Platelets consist of many activated growth factors that could advance bone regeneration. However, it gets exhausted within 24 hours, if delivered directly to the site, which prevents it from performing its role in regeneration [20, 27]. Hence, a proper delivery system is essential for applying platelets. Our results showed that the combination of PRP and MSCs significantly increased the osteogenic differentiation and proliferation, which can be reasonably explained since PRP consists primarily of PDGF, IGF and TGF-beta, which promote osteoblast proliferation and maturation [27]. Moreover, PRP can be derived from whole blood and is biocompatible to the cells.

In this study, by transplanting BMP2-modified MSCs with nCS and PRP fibrin gel into well-established critical-sized rat calvarial bone defects, we found that the BMD of this group was much higher than that in other groups and exhibited dramatic bone repair in bone defects at 8 weeks following the surgery. Histological analysis complemented the BMD result and all defects were almost covered with newly- mineralized bone tissue. These results demonstrated that the combination of BMP2-modified MSCs with an nCS/PRP gel scaffold is an effective approach for bone regeneration and repair.

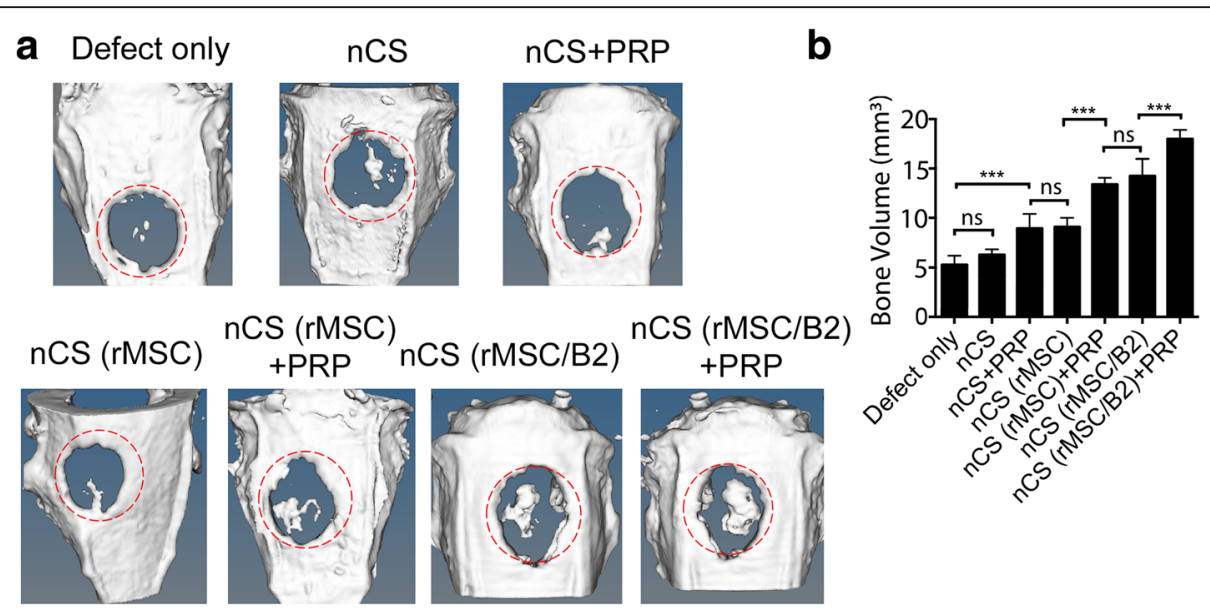

Fig. 5 Micro-CT analysis of newly formed bone: a Images of calvarial bone obtained using micro-CT, 8 weeks after surgery. Higher amount of bone was detected in the nCS (rMSC/B2) + PRP group as compared to other groups. b Quantitative analysis of bone volume in the implanted area from $A$. The average bone volume were analyzed $(n=4) .{ }^{* *} p<0.01 ;{ }^{* * *} p<0.001$; $n s:$ not statistically significant. B2 bone morphogenetic protein 2, MSCS mesenchymal stem cells, nCS nano-calcium sulfate, PRP platelet-rich plasma 
Notably, the critical-sized defects were repaired much better in the MSCs-containing groups compared with the group without MSCs, and exhibited no sign of rejection in all groups, indicating that most of the implanted allogeneic MSCs maintained their viability and had no apparent immunorejection by the host. This conclusion was supported by some previous findings. For example, $\mathrm{Li}$ et al. [28] found that there is no rejection and/or graft versus host disease by intravenously injecting green fluorescent protein-labeled allogeneic MSCs into rabbits. Saito et al. [29] demonstrated that the rats display immunological tolerance to mouse MSCs.

Recombinant BMP2 has to be present in high concentrations (up to milligrams) in order to induce an osteogenic effect, and clinical applications are limited to a single dose at the time of implantation. While the use of lentivirus and retrovirus vector has disadvantages including gene integration into genomic DNA, which could lead to the mutation, and life-time and uncontrolled ectopic expression of the target genes, likely causing tumor or other severe side effects. Therefore, we used a recombinant adenoviral vector carrying human $B M P 2$ gene (Adv-BMP2) to transduce MSCs in order to prolong the BMP2 activity [30]. Our previous study showed that adenovirus-mediated BMP2 expression lasts up to 14 days, which is sufficient to stimulate bone regeneration and repair [15]. Here, besides the results that support the concept that BMP2-engineered MSCs enhance bone regeneration, the effects of PRP and BMP2 delivery in MSCs observed in this study highlights the importance of BMP2, PRP, and MSCs in bone healing. When PRP is used in conjunction with MSCs in vitro, the findings demonstrated that it is inclined toward promoting the cellular proliferation of MSCs [31]. Although PRP also consists of a small amount of BMP2, however, the increased proliferative activity may lead to altered morphology of the cell distribution as well as poor differentiation [32, 33]. BMP2 stimulates the differentiation and maturation of MSCs [34]. When ectopic BMP2 was used in conjunction with PRP, we observed that PRP could synergistically promote the activity of BMP2 thus increasing the proliferation activity and the osteogenic potential of MSCs [32, 33].

\section{Conclusions}

In summary, this study provides the first evidence to support that this novel delivery system of nCS/PRP sandwich-like scaffold combined with MSCs/B2 strongly stimulates bone formation. This system can be useful not only in critical-sized bone defects, but also in defects with limited accessibility, such as in repairing periodontal bone defects. Its use in minimally invasive techniques, such as in situ fracture fixation and percutaneous vertebroplasty to fill the lesions and strengthen osteoporotic bone, is another area to pursue further.

\section{Additional file}

Additional file 1: Figure S1. PRP and scaffold preparation. (A) PRP preparation by two times centrifuge. PRP platelet-rich plasma, PPP platelet-poor plasma. (B) Schematic of nCS/PRP scaffold preparation, and MSCs loading. nCS nano-calcium sulfate, B2 BMP2. (C) Representative images of nCS disks before and after PRP loading. 8-mm defect was created in rat calvaria bone, and scaffolds were put into the defects. (TIF 2456 kb)

\begin{abstract}
Abbreviations
ALP: Alkaline phosphatase; BMPs: Bone morphogenetic proteins; GFs: Growth factors; IGF: Insulin-like growth factor; MSCs: Mesenchymal stem cells; nCS: Nano-calcium sulfate; PDGF: Platelet-derived growth factor; PPP: platelet-poor plasma; PRP: Platelet-rich plasma; TGF-beta: Transforming growth factors beta
\end{abstract}

\section{Acknowledgements}

Not applicable

Funding

Research reported in this publication was supported by the National Institute of Dental and Craniofacial Research and the National Institute of Aging, part of the National Institutes of Health, under Award Numbers DE023105 and AG048388 to SY.

\section{Availability of data and materials}

None

Authors' contributions

$\mathrm{ZL}, \mathrm{XY}, \mathrm{RD}$, and SY contributed to the study design, study performance, and preparation of the manuscript. GF contributed to data analysis and interpretation, and preparation and revision of the manuscript. Cl, CL, CW contributed to the study performance. ZL, XY, GF, Cl and SY contributed to the data analysis and interpretation and revision of the manuscript. All authors read and approved the final manuscript.

\section{Competing interests}

The authors declare that they have no competing interests.

Consent for publication

Not applicable

Ethics approval and consent to participate

The protocol was reviewed and approved by the University at Buffalo Animal Care and Use Committee.

\section{Publisher's Note}

Springer Nature remains neutral with regard to jurisdictional claims in published maps and institutional affiliations.

\section{Author details}

${ }^{1}$ Department of Oral Biology, School of Dental Medicine, University of Buffalo, The State University of New York, Buffalo, NY, USA. ²Developmental Genomics Group, New York State Center of Excellence in Bioinformatics and Life Sciences, University of Buffalo, The State University of New York, Buffalo, NY, USA. ${ }^{3}$ Department of Anatomy and Cell Biology, School of Dental Medicine, University of Pennsylvania, Philadelphia, PA 19104, USA. ${ }^{4}$ Department of Orthopedics, Fourth Affiliated Hospital, China Medical University, Shenyang, China. ${ }^{5}$ Departments of Biomedical Engineering and Neurosurgery, Toshiba Stroke And Vascular Research Center, University of Buffalo, The State University of New York, Buffalo, NY, USA.

Received: 14 October 2016 Revised: 7 February 2017 Accepted: 5 May 2017 Published online: 25 May 2017

\section{References}

1. Glidear J. Bone grafts: a review of the literature. J Foot Surg. 1977;16:146-8.

2. Rose LF, Rosenberg E. Bone grafts and growth and differentiation factors for regenerative therapy: a review. Pract Proced Aesthet Dent. 2001;13:725-34. quiz $736,721-722$ 
3. Kurz LT, Garfin SR, Booth Jr RE. Harvesting autogenous iliac bone grafts. A review of complications and techniques. Spine (Phila Pa 1976). 1989;14:1324-31.

4. Bruder SP, Fink DJ, Caplan Al. Mesenchymal stem cells in bone development, bone repair, and skeletal regeneration therapy. J Cell Biochem. 1994;56:283-94.

5. Kraus KH, Kirker-Head C. Mesenchymal stem cells and bone regeneration. Vet Surg. 2006;35:232-42.

6. Fernandes $\mathrm{G}$, Yang S. Application of platelet-rich plasma with stem cells in bone and periodontal tissue engineering. Bone Res. 2016;4:16036.

7. Mackenzie TC, Flake AW. Multilineage differentiation of human MSC after in utero transplantation. Cytotherapy. 2001;3:403-5.

8. Gregory CA, Ylostalo J, Prockop DJ. Adult bone marrow stem/progenitor cells (MSCs) are preconditioned by microenvironmental "niches" in culture: a two-stage hypothesis for regulation of MSC fate. Sci STKE. 2005;2005:pe37.

9. Zhuang H, Zhang X, Zhu C, Tang X, Yu F, Shang GW, Cai X. Molecular mechanisms of PPAR-gamma governing MSC osteogenic and adipogenic differentiation. Curr Stem Cell Res Ther. 2016;11:255-64.

10. Vogl M, Fischer J, Jager M, Zilkens C, Krauspe R, Herten M. Can thrombinactivated platelet releasate compensate the age-induced decrease in cell proliferation of MSC? J Orthop Res. 2013;31:1786-95.

11. Hao J, Zhang Y, Jing D, Shen Y, Tang G, Huang S, Zhao Z. Mechanobiology of mesenchymal stem cells: Perspective into mechanical induction of MSC fate. Acta Biomater. 2015;20:1-9.

12. Shah $P$, Keppler L, Rutkowski J. Bone morphogenic protein: an elixir for bone grafting-a review. J Oral Implantol. 2012;38:767-78.

13. Kuboki $Y$, Sasaki M, Saito A, Takita H, Kato H. Regeneration of periodontal ligament and cementum by BMP-applied tissue engineering. Eur J Oral Sci. 1998;106 Suppl 1:197-203.

14. He X, Dziak R, Yuan X, Mao K, Genco R, Swihart M, Sarkar D, Li C, Wang C, Lu L, et al. BMP2 genetically engineered MSCs and EPCs promote vascularized bone regeneration in rat critical-sized calvarial bone defects. PLoS One. 2013:8:e60473.

15. He X, Dziak R, Mao K, Genco R, Swihart M, Li C, Yang S. Integration of a novel injectable nano calcium sulfate/alginate scaffold and BMP2 genemodified mesenchymal stem cells for bone regeneration. Tissue Eng Part A. 2013;19:508-18

16. Mautner K, Malanga GA, Smith J, Shiple B, Ibrahim V, Sampson S, Bowen JE. A call for a standard classification system for future biologic research: the rationale for new PRP nomenclature. PM R. 2015;7:S53-59.

17. Rodrigues SV, Acharya AB, Thakur SL. Platelet-rich plasma. A review. N Y State Dent J. 2012;78:26-30.

18. Arora NS, Ramanayake T, Ren YF, Romanos GE. Platelet-rich plasma: a literature review. Implant Dent. 2009;18:303-10.

19. Nikolidakis D, Jansen JA. The biology of platelet-rich plasma and its application in oral surgery: literature review. Tissue Eng Part B Rev. 2008;14: 249-58.

20. Isaac C, Gharaibeh B, Witt M, Wright VJ, Huard J. Biologic approaches to enhance rotator cuff healing after injury. J Shoulder Elbow Surg. 2012;21: 181-90.

21. Han X, Liu H, Wang D, Su F, Zhang Y, Zhou W, Li S, Yang R. Alveolar bone regeneration around immediate implants using an injectable nHAC/CSH loaded with autogenic blood-acquired mesenchymal progenitor cells: an experimental study in the dog mandible. Clin Implant Dent Relat Res. 2013; 15:390-401.

22. Landesberg R, Burke A, Pinsky D, Katz R, Vo J, Eisig SB, Lu HH. Activation of platelet-rich plasma using thrombin receptor agonist peptide. J Oral Maxillofac Surg. 2005;63:529-35.

23. Huang S, Wang Z. Influence of platelet-rich plasma on proliferation and osteogenic differentiation of skeletal muscle satellite cells: an in vitro study. Oral Surg Oral Med Oral Pathol Oral Radiol Endod. 2010;110:453-62.

24. Park YB, Mohan K, Al-Sanousi A, Almaghrabi B, Genco RJ, Swihart MT, Dziak R. Synthesis and characterization of nanocrystalline calcium sulfate for use in osseous regeneration. Biomed Mater. 2011;6:055007.

25. Hammouche $\mathrm{S}$, Khan W, Drouin $\mathrm{H}$, Procter $\mathrm{H}$, McNicholas M. Calcium salts bone regeneration scaffolds: a review article. Curr Stem Cell Res Ther. 2012; 7:336-46.

26. Thomas MV, Puleo DA, Al-Sabbagh M. Calcium sulfate: a review. J Long Term Eff Med Implants. 2005;15:599-607.

27. Lu HH, Vo JM, Chin HS, Lin J, Cozin M, Tsay R, Eisig S, Landesberg R. Controlled delivery of platelet-rich plasma-derived growth factors for bone formation. J Biomed Mater Res A. 2008;86:1128-36.
28. Li ZH, Liao W, Cui XL, Zhao Q, Liu M, Chen YH, Liu TS, Liu NL, Wang F, Yi Y, Shao NS. Intravenous transplantation of allogeneic bone marrow mesenchymal stem cells and its directional migration to the necrotic femoral head. Int J Med Sci. 2011;8:74-83.

29. Saito T, Kuang JQ, Bittira B, Al-Khaldi A, Chiu RC. Xenotransplant cardiac chimera: immune tolerance of adult stem cells. Ann Thorac Surg. 2002;74: 19-24. discussion 24

30. Sharma S, Sapkota D, Xue Y, Sun Y, Finne-Wistrand A, Bruland O, Mustafa K. Adenoviral mediated expression of BMP2 by bone marrow stromal cells cultured in 3D copolymer scaffolds enhances bone formation. PLOS One. 2016:11:e0147507.

31. Roubelakis MG, Trohatou O, Roubelakis A, Mili E, Kalaitzopoulos I, Papazoglou G, Pappa Kl, Anagnou NP. Platelet-rich plasma (PRP) promotes fetal mesenchymal stem/stromal cell migration and wound healing process. Stem Cell Rev. 2014:10:417-28.

32. Xie X, Zhang C, Tuan RS. Biology of platelet-rich plasma and its clinical application in cartilage repair. Arthritis Res Ther. 2014;16:204.

33. Jiang $Z Q$, Liu HY, Zhang LP, Wu ZQ, Shang DZ. Repair of calvarial defects in rabbits with platelet-rich plasma as the scaffold for carrying bone marrow stromal cells. Oral Surg Oral Med Oral Pathol Oral Radiol. 2012;113:327-33.

34. Rubio-Azpeitia E, Andia I. Partnership between platelet-rich plasma and mesenchymal stem cells: in vitro experience. Muscles Ligaments Tendons J. 2014;4:52-62

\section{Submit your next manuscript to BioMed Central and we will help you at every step:}

- We accept pre-submission inquiries

- Our selector tool helps you to find the most relevant journal

- We provide round the clock customer support

- Convenient online submission

- Thorough peer review

- Inclusion in PubMed and all major indexing services

- Maximum visibility for your research

Submit your manuscript at www.biomedcentral.com/submit

) BioMed Central 